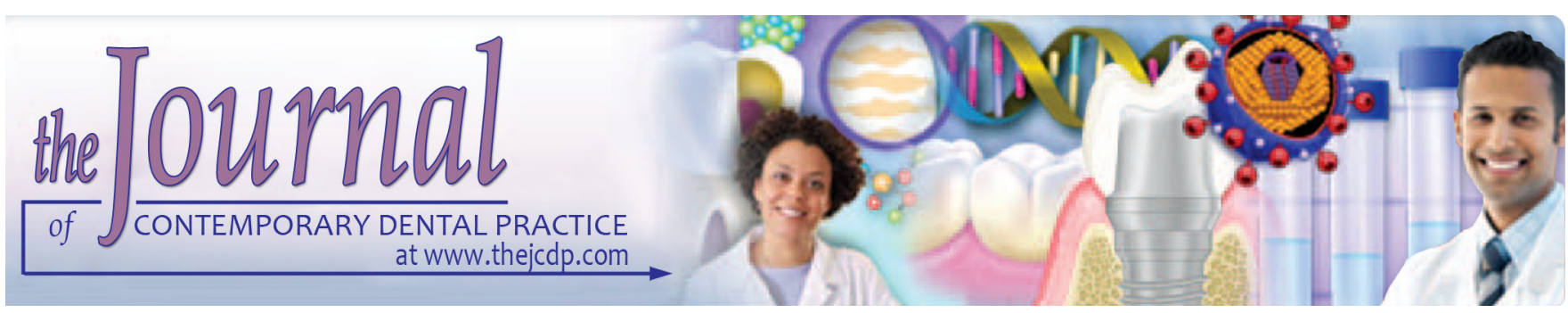

\title{
Evaluation of Soft Tissue Landmark Reliability between Manual and Computerized Plotting Methods
}

\author{
${ }_{1}^{1}$ Geetha Kasinathan, ${ }^{2}$ Pradeep B Kommi, ${ }^{3}$ Senthil M Kumar, ${ }^{4}$ Aniruddh Yashwant, ${ }^{5}$ Nandakumar Arani, ${ }^{6}$ Senkutvan Sabapathy
}

\begin{abstract}
Aim: The aim of the study is to evaluate the reliability of soft tissue landmark identification between manual and digital plottings in both $X$ and $Y$ axes.

Materials and methods: A total of 50 pretreatment lateral cephalograms were selected from patients who reported for orthodontic treatment. The digital images of each cephalogram were imported directly into Dolphin software for onscreen digitalization, while for manual tracing, images were printed using a compatible X-ray printer. After the images were standardized, and 10 commonly used soft tissue landmarks were plotted on each cephalogram by six different professional observers, the values obtained were plotted in $\mathrm{X}$ and $\mathrm{Y}$ axes. Intraclass correlation coefficient was used to determine the intrarater reliability for repeated landmark plotting obtained by both the methods.

Results: The evaluation for reliability of soft tissue landmark plottings in both manual and digital methods after subjecting it to interclass correlation showed a good reliability, which was nearing complete homogeneity in both $X$ and $Y$ axes, except for $Y$ axis of throat point in manual plotting, which showed moderate reliability as a cephalometric variable. Intraclass correlation of soft tissue nasion had a moderate reliability along $X$ axis. Soft tissue pogonion shows moderate reliability in $Y$ axis. Throat point exhibited moderate reliability in $\mathrm{X}$ axis.
\end{abstract}

Conclusion: The interclass correlation in $\mathrm{X}$ and $\mathrm{Y}$ axes shows high reliability in both hard tissue and soft tissue except for throat point in $Y$ axis, when plotted manually.

The intraclass correlation is more consistent and highly reliable for soft tissue landmarks and the hard tissue landmark identification is also consistent.

Clinical significance: The results obtained for manual and digital methods were almost similar, but the digital landmark plotting has

\footnotetext{
${ }^{1-4,6}$ Department of Orthodontics and Dentofacial Orthopedics Indira Gandhi Institute of Dental Sciences, Sri Balaji Vidyapeeth University, Puducherry, India

${ }^{5}$ Department of Orthodontics and Dentofacial Orthopedics Meenakshi Ammal Dental College and Hospital, Chennai, Tamil Nadu, India

Corresponding Author: Geetha Kasinathan, Department of Orthodontics and Dentofacial Orthopedics, Indira Gandhi Institute of Dental Sciences, Puducherry, India, e-mail: 21gaugewire@ gmail.com
}

an added advantage in archiving, retrieval, transmission, and can be enhanced during plotting of lateral cephalograms. Hence, the digital method of landmark plotting could be preferred for both daily use and research because of the advantages.

Keywords: Cephalometric, Dolphin software, Intraclass correlation, Reliability.

How to cite this article: Kasinathan G, Kommi PB, Kumar SM, Yashwant A, Arani N, Sabapathy S. Evaluation of Soft Tissue Landmark Reliability between Manual and Computerized Plotting Methods. J Contemp Dent Pract 2017;18(4):317-321.

Source of support: Nil

Conflict of interest: None

\section{INTRODUCTION}

In orthodontics, cephalometrics plays a crucial role in diagnosis and treatment planning. Cephalometric radiograph is the product of a two-dimensional image of the skull in lateral view, which helps in enabling the relationship between teeth, bone, soft tissue, and empty spaces in horizontal and vertical planes of space. It also helps in evaluation, diagnosis, treatment results, and prediction of growth.

The era of radiographic cephalometry began in orthodontics in 1931 by Broadbent, ${ }^{1}$ who happened to simultaneously present a standardized cephalometric technique for obtaining standardized radiographs of the head.

The major sources of errors in cephalometric analysis are radiographic film magnification, tracing, measuring, and landmark identification. The inconsistency in landmark identification may lead to major errors in cephalometric analysis.

"Dolphin" cephalometric software, which according to manufacturer, promises accurate landmark identification, cephalometric analysis, treatment prediction in orthognathic cases, superimposition, and can act as a tool for educating the patient on treatment outcome.

Several studies have been undertaken to compare the accuracy of landmark identification in scanned or 
digitized lateral cephalogram with the manual methods, whereas studies evaluating the reliability in landmark identification of digitally obtained radiographs with the manual method are scanty in the literature.

Hence, the objective of this study is to evaluate the errors and reliability in cephalometric landmark identification using Dolphin orthodontic software, which is commercially available in the market for cephalometric analysis, and compare it with the manual cephalometric landmark identification.

\section{Aims and Objectives}

\section{Aim}

The aim of the study is to detect errors and reliability of landmark identification between manual and digital landmark plottings for soft tissue landmarks.

\section{Objective}

The objective of the study is to conclude on the superior method of plotting cephalometric landmarks and their reliability between manual and computerized methods (Dolphin software) for soft tissue landmark points.

\section{MATERIALS AND METHODS}

A total of 50 pretreatment digital lateral cephalograms of patients, who reported to our department for orthodontic consultation and treatment, were taken using digital cephalometer (Orthophos Xg-Sirona Model No.: D3352) and a written consent form was obtained from all the patients. The criteria for selecting the 50 cephalograms were as follows:

- Good-quality lateral cephalograms with sufficient contrast to permit proper identification of the landmarks

- Presence of permanent dentition with no missing and impacted teeth that would have hindered the landmark identification

- Patients without trauma, syndromes, craniofacial deformity, or gross asymmetry

- Lateral cephalograms of patients between 18 and 25 years of age

- Lateral cephalograms obtained in natural head position. $^{2}$

The originally saved digital cephalographic images are retrieved from the computer in which they were stored. As suggested by Jacobson, ${ }^{3}$ among the 50 selected images, three registration crosses for orientation were marked, two in cranium and one in cervical vertebrae region for reorientation and two fiduciary points were chosen on the rulers that were images with the patients. The $\mathrm{Y}$ axis was constructed by the software connecting the two fiduciary points as the vertical reference for landmark coordination, the $\mathrm{X}$ axis was constructed perpendicular to this line that served as horizontal reference, ${ }^{4}$ and they were printed to $100 \%$ of the original size. The prints were obtained in Fuji Medical Dry Imaging Film of size $20 \times$ $25 \mathrm{~cm}(8 \times 10$ inches $) .^{5}$

These printed lateral cephalograms were subjected to manual landmark plotting, and the digital images cephalograms were imported to computer-aided cephalometric software Dolphin imaging V.11.8 to perform the landmark plotting.

A total number of 10 soft tissue landmarks (Fig. 1) were identified by six qualified investigators; each observer was to perform landmark plotting per each cephalogram three times manually and three times digitally, at a 2 weeks interval as performed by Yu et al. ${ }^{6}$ These landmarks play a significant role in routine orthodontic diagnosis and treatment planning. Not more than two radiographs were plotted at a given time to avoid errors due to operator fatigue in both the methods.

\section{Manual Plotting}

The entire 50 samples ${ }^{6,7}$ were manually plotted in a dark room over an X-ray view box (Fig. 2) on acetate sheet of thickness $0.003^{\prime \prime 7-9}$ with a $0.5 \mathrm{~mm}^{8,10}$ lead pencil by six qualified investigators. All tracing sheets were plotted and each landmark was separately transferred to graph sheet and later the values of the landmarks were evaluated in $\mathrm{X}$ and $\mathrm{Y}$ axes. ${ }^{8}$

\section{Digital Plotting}

Digital plotting of all 10 landmarks was performed by the same six investigators using Dolphin imaging V.11.8

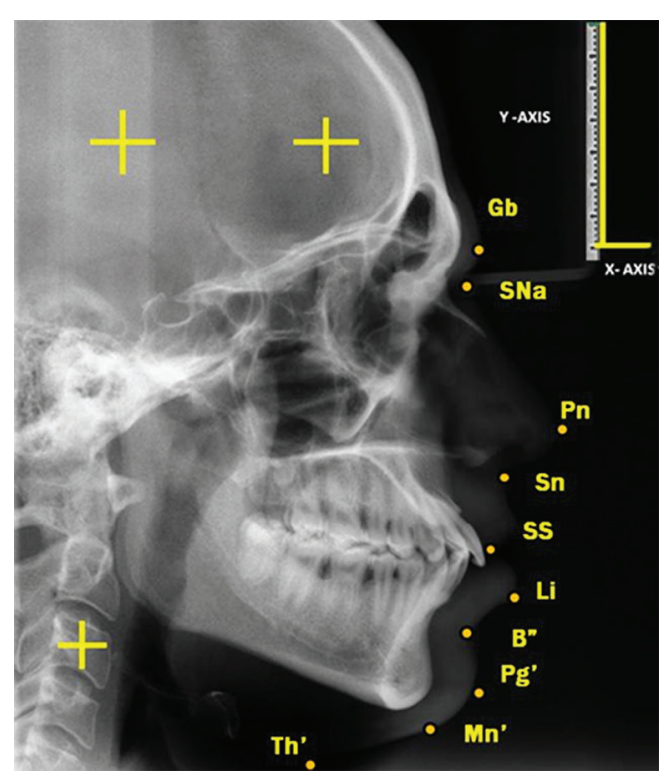

Fig. 1: Soft tissue landmarks, Gb: Glabella, S: Na-soft tissue nasion, Pn: Pronasale, Sn: Subnasale, SS: Stomium superius, Li: Labrale Infereus, B': Soft tissue point B, Pg': Soft tissue Pogonion, Mn': Soft tissue menton, Th': Throat point 


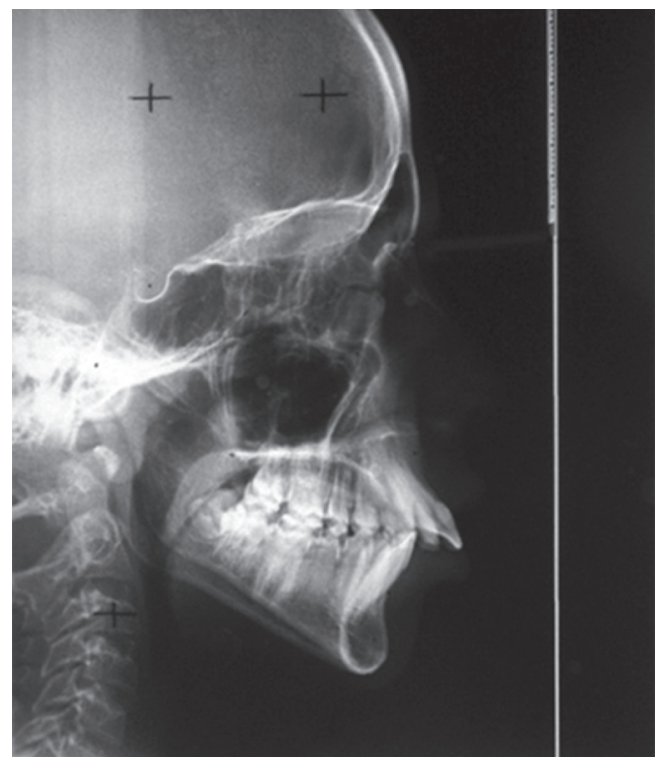

Fig. 2: Manual plotting over an X-ray view box

software. The images were calibrated by dpi settings and viewed in a $15^{\prime \prime}$ liquid crystal display flat screen monitor (Fig. 3). The landmarks were manually identified using cursor controlled mouse. ${ }^{8}$ After plotting each, cephalograms were printed in $20 \times 25 \mathrm{~cm}$ ( $8 \times 10$ inches) and landmarks were transferred to the graph sheet to get values in $\mathrm{X}$ and $\mathrm{Y}$ axes. ${ }^{8}$

\section{Statistical Analysis}

A total of 12,000 values were obtained of which were $6000 \mathrm{X}$ component and $6000 \mathrm{Y}$ component. It includes 600 readings for an individual landmark. The analysis was carried out using Statistical Package for Social Sciences version 16.0. Average values of each landmark plotting were calculated and presented as mean \pm standard deviation. Differences in mean were analyzed using analysis of variances (Table 1). The level of significance was set at $\mathrm{p}<0.05$. Intraclass correlation coefficient (ICC) is used to determine the intrarater reliability for both the techniques. According to Landis and Koch, ${ }^{11}$ the following ICC interpretation

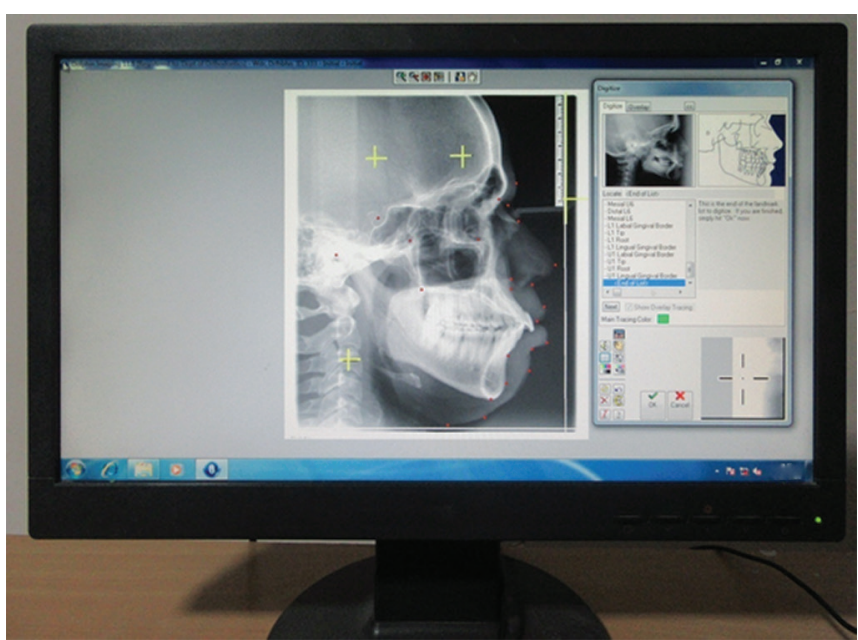

Fig. 3: Landmark plotting with Dolphin imaging V.11.8 (Dolphin imaging and management solutions, Chatsworth, CA 91311) software

scale was used: Poor to fair (below 0.4), moderate (0.41$0.60)$, excellent $(0.61-0.80)$, and almost perfect $(0.81-1)$.

\section{DISCUSSION}

Evaluation of errors in landmark identification in both manual and digital methods for soft tissue anatomical points had been performed, but there is little or no research done to identify the errors for soft tissue landmark points to authenticate or compare our results with already existing research. According to Agarwal et al, ${ }^{9}$ very few studies in the literature have been evaluated on soft tissue measurements due to the uncertainty in identifying soft tissue landmarks. Soft tissue cephalometric analysis is gaining popularity, as the orthodontists have understood the importance of soft tissues and their influence on diagnosis and treatment planning.

As per Table 2, ICC value of glabella in X axis is near homogeneity. However, when manual plotting is directly compared with cephalometric plotting, the ICC is 0.805 indicating good reliability. Moreover, in Y axis, it is 0.980 in manual identification and 0.994 in digital identification. Digital plotting has a higher intraclass correlation value

Table I: Results Obtained By Manual And Dolphin Plotting Of Soft Tissue Landmarks

\begin{tabular}{|c|c|c|c|c|c|c|c|c|}
\hline \multirow{2}{*}{$\begin{array}{l}\text { Softtissue } \\
\text { Landmarks }\end{array}$} & \multicolumn{2}{|c|}{ Manual Mean \pm S.D } & \multicolumn{2}{|c|}{ Dolphin Mean SD } & \multicolumn{2}{|c|}{ Manual ICC } & \multicolumn{2}{|c|}{ Dolphin ICC } \\
\hline & $x$ & $Y$ & $x$ & $Y$ & $x$ & $Y$ & $x$ & $Y$ \\
\hline Glabella & $33.85 \pm 6.79$ & $9.62 \pm 7.982$ & $33.68 \pm 6.19$ & $8.359 \pm 7.378$ & 0.941 & 0.980 & 0.995 & 0.994 \\
\hline S. Nasion & $37.27 \pm 5.18$ & $22.90 \pm 5.5$ & $37.65 \pm 4.84$ & $21.81 \pm 5.16$ & 0.909 & 0.787 & 0.994 & 0.989 \\
\hline Pronasale & $15.93 \pm 13.11$ & $60.50 \pm 12.5$ & $14.55 \pm 12.10$ & $58.79 \pm 12.61$ & 0.992 & 0.981 & 0.992 & 0.999 \\
\hline Sub.nasale & $28.99 \pm 12.97$ & $69.68 \pm 17.2$ & $27.60 \pm 11.43$ & $71.82 \pm 11.5$ & 0.929 & 0.845 & 0.999 & 0.954 \\
\hline Stomium superius & $32.59 \pm 17.28$ & $89.47 \pm 22.5$ & $30.05 \pm 15.96$ & $92.25 \pm 15.51$ & 0.781 & 0.990 & 0.999 & 0.998 \\
\hline Labrale Inferius & $32.27 \pm 22.63$ & $105.06 \pm 19.9$ & $28.76 \pm 19.10$ & $106.1 \pm 18.27$ & 0.992 & 0.832 & 0.999 & 0.997 \\
\hline Soft B & $38.72 \pm 20.54$ & $108.94 \pm 23.0$ & $36.29 \pm 17.78$ & $110.61 \pm 18.5$ & 0.919 & 0.865 & 0.998 & 0.947 \\
\hline S. Pog & $46.74 \pm 23.94$ & $114.72 \pm 28.3$ & $57.40 \pm 38.58$ & $103.2 \pm 45.04$ & 0.709 & 0.922 & 0.963 & 0.975 \\
\hline S. Ment & $59.29 \pm 23.92$ & $136.76 \pm 31.0$ & $58.78 \pm 23.95$ & $137.7 \pm 25.64$ & 0.954 & 0.962 & 0.998 & 0.995 \\
\hline Throat Point & $93.04 \pm 17.68$ & $142.25 \pm 29.2$ & $89.37 \pm 16.01$ & $144.44 \pm 16.1$ & 0.926 & 0.466 & 0.963 & 0.994 \\
\hline
\end{tabular}


Table 2: Results Obtained On Comparing ICC Values Of SoftTissue Landmark Plotting Between Manual \& Computerised Method

\begin{tabular}{|c|c|c|c|c|c|c|}
\hline \multirow[b]{2}{*}{ Landmarks } & \multicolumn{2}{|c|}{ ICC-manual } & \multicolumn{2}{|c|}{$\begin{array}{l}\text { ICC- } \\
\text { computerised }\end{array}$} & \multicolumn{2}{|c|}{$\begin{array}{l}\text { ICC- } \\
\text { Manual vs } \\
\text { computerised }\end{array}$} \\
\hline & $x$ & $Y$ & $x$ & $Y$ & $x$ & $Y$ \\
\hline Glabella & 0.941 & 0.980 & 0.995 & 0.994 & 0.805 & 0.780 \\
\hline S.nasion & 0.909 & 0.787 & 0.994 & 0.989 & 0.841 & 0.425 \\
\hline Pronasale & 0.992 & 0.981 & 0.992 & 0.999 & 0.732 & 0.764 \\
\hline Sub.nasale & 0.929 & 0.845 & 0.999 & 0.954 & 0.751 & 0.639 \\
\hline Stomium Superius & 0.781 & 0.990 & 0.999 & 0.998 & 0.726 & 0.560 \\
\hline Labrale Inferius & 0.992 & 0.832 & 0.999 & 0.997 & 0.649 & 0.882 \\
\hline Soft Tissue Point B & 0.919 & 0.865 & 0.998 & 0.947 & 0.732 & 0.730 \\
\hline S. Pog & 0.709 & 0.922 & 0.963 & 0.975 & 0.456 & 0.849 \\
\hline S. Ment & 0.954 & 0.962 & 0.998 & 0.995 & 0.759 & 0.765 \\
\hline Throat Point & 0.926 & 0.466 & 0.963 & 0.994 & 0.643 & 0.483 \\
\hline
\end{tabular}

than manual plotting method and both values are near homogeneity. Moreover, in comparison of both manual and digital techniques, the ICC value is 0.780 , which indicates perfect agreement for cephalometric reliability.

Soft tissue nasion, when assessed in $\mathrm{X}$ axis in manual and digital landmark methods, it denotes high homogeneity. When both the techniques are directly compared, the intraclass correlation value is 0.841 , which indicates a complete homogeneity. Along Y axis, digital ICC value is higher than manual ICC value; both the values are near homogeneity. On direct comparison of both the techniques, ICC value is 0.425 , which indicates moderate reliability.

Pronasale, when evaluated in $\mathrm{X}$ axis, has an intraclass correlation of 0.992 manually and 0.992 in digital. Moreover, when both are directly compared, the ICC is 0.732 , which indicates excellent reliability. In Y axis, in manual and computerized plottings it is indicating complete homogeneity, but when compared directly, the value is 0.764 indicating excellent reliability of cephalometric variable.

Subnasale with ICC in $\mathrm{X}$ axis in both manual and digital plottings denotes complete homogeneity. When directly compared between manual and computerized methods, ICC is 0.751 indicating excellent reliability. Manual plotting in $\mathrm{Y}$ axis is 0.845 and 0.954 in digital method, indicating good reliability; when directly compared, ICC was 0.639 , denoting good reliability for cephalometric variables.

Stomion superius with ICC value in $\mathrm{X}$ axis indicates complete homogeneity in digital method and denoting good reliability in manual method. When compared directly between manual and digital, ICC value indicates excellent agreement of cephalometric variable. Along $\mathrm{Y}$ axis, ICC in both the techniques indicates complete homogeneity. On comparing both manual and digital landmark plotting methods, the ICC value is 0.560 , denoting moderate agreement as a cephalometric variability.
The soft tissue landmark labrale inferius in $\mathrm{X}$ axis has an ICC value showing good reliability. Along Y axis, ICC in manual and digital plotting methods indicate complete homogeneity. On direct comparison between both the methods, the ICC value is 0.882 , which shows complete homogeneity.

Soft tissue point B with ICC values in manual and digital method, are near to one; it denotes high homogeneity. When digital and manual landmark plotting techniques are compared, ICC value is 0.732 indicating excellent reliability. On assessing along Y axis, ICC value indicates almost perfect reliability. However, when compared directly between both the techniques, ICC is 0.730 , denoting excellent reliability for cephalometric variables.

The ICC value of soft tissue pogonion in $X$ axis in manual plotting method indicates good reliability, and in digital plotting method, it denotes complete homogeneity. On direct comparison of both techniques, the ICC is 0.456 , indicating moderate agreement. Along Y axis, ICC indicates complete homogeneity. On direct comparison, the ICC value indicates complete homogeneity.

Soft tissue menton with ICC value in $\mathrm{X}$ axis indicates complete homogeneity. On direct comparison between both the methods, the ICC value is 0.759 , indicating good agreement. Along Y axis, the ICC value in manual plotting indicates complete homogeneity. On direct comparison of both methods, ICC value is 0.765 , indicating good reliability for cephalometric variable.

The soft tissue landmark throat point in $\mathrm{X}$ axis in manual and in digital landmark plottings indicate high homogeneity. On direct comparison between both the techniques, intraclass correlation is 0.643 , denoting excellent agreement. On Y axis, the manual ICC value denotes moderate reliability, whereas digital ICC value denotes complete homogeneity. On comparison, the ICC value indicates moderate reliability.

Lim and Foong ${ }^{12}$ in the British Journal of Orthodontics 1997, have suggested in their study that lower lip was only statistically significant in Y axis and other landmarks like glabella, pronasale, and soft tissue pogonion had a greater reliability in $X$ axis. Glabella and soft tissue pogonion had a mean random error of more than $1 \mathrm{~mm}$ in $Y$ axis because of inability to define one precise point of identification for a particular landmark, resulting in a dispersion of identification points along the soft tissue contour irrespective of image quality.

Nikneshan et $\mathrm{al}_{1}^{13}$ have stated that soft tissue landmarks, such as soft tissue menton, are reliable in both $X$ and $Y$ axes in nonenhanced radiograph, and showed less reliability in $\mathrm{Y}$ axis on enhancement. The reliability of labrale inferius in $X$ axis was decreased on enhanced and increased in nonenhanced radiograph. Subnasale and pronasale showed increased reliability in X axis, whereas 
pogonion showed decreased reliability along $\mathrm{Y}$ axis in enhanced method.

Cooke and Wei ${ }^{14}$ have stated that lip prominence points are poor landmarks to identify.

Tikku et $\mathrm{l}^{15}$ have stated that soft tissue landmarks like labrale inferius, stomion superius, soft tissue menton, soft tissue pogonion, and subnasale are difficult to locate in manual technique, since they are located on contoured area.

Dvortsin et $\mathrm{al}^{16}$ have stated that stomion was the least reproducible, since some subjects had their lips in contact, while in others, the lips were slightly apart when relaxed. Moreover, it is difficult to define particularly in subjects with lips-apart posture where the lowest dependent point on the upper lip (stomion superius) has to be estimated.

The evaluation for reliability of soft tissue landmark plotting in both manual and digital plotting methods after subjecting it to interclass correlation showed a good reliability, which was nearing complete homogeneity in both $X$ and $Y$ axes, except $Y$ axis of throat point in manual plotting method, which showed moderate reliability as a cephalometric variable. Intraclass correlation of soft tissue nasion and throat point exhibited moderate reliability in $\mathrm{Y}$ axis, whereas soft tissue pogonion shows moderate reliability in $\mathrm{X}$ axis when intraclass correlation was performed for both manual and computerized landmark identifications.

\section{CONCLUSION}

To conclude the study for evaluating the reliability of landmark identification in manual and digital methods.

The interclass correlation in $X$ and $Y$ axes shows high reliability in soft tissue except throat point in $\mathrm{Y}$ axis, when plotted manually, which has moderate reliability.

The intraclass correlation is more consistent and highly reliable for soft tissue landmarks.

The results obtained for manual and digital were almost similar, but the digital landmark plotting has an added advantage in archiving, retrieval, transmission, and can be enhanced during plotting of lateral cephalograms, so that the digital method of landmark plotting could be preferred for both daily use and research because of the advantages.

\section{REFERENCES}

1. Broadbent $\mathrm{BH}$. A new X-ray technique and its application to orthodontia. Angle Orthod 1931 Apr;1(2):45-66.
2. Prabhakar P, Rajakumar P, Karthikeyan MK, Saravanan R, Vikram NR, Reddy A. A hard tissue cephalometric comparative study between hand tracing and computerized tracing. J Pharm Bioallied Sci 2014 Jul;(6Suppl 1):S101-S106.

3. Jacobson, A. Radiographic cephalometry: from basics to video imaging. Chicago: Quintessence Publishing Co.; 1995. p. 336.

4. Chang ZC, Hu FC, Lai E, Yao CC, Chen MH, Chen YJ. Landmark identification errors on cone-beam computed tomography-derived cephalograms and conventional digital cephalograms. Am J Orthod Dentofacial Orthop 2011 Dec;140(6):e289-e297.

5. Mahto RK, Kharbanda OP, Duggal R, Sardana HK. A comparison of cephalometric measurements obtained from two computerized cephalometric softwares with manual tracings. J Indian Orthod Soc 2016 Jan;50(3):162-170.

6. Yu SH, Nahm DS, Baek SH. Reliability of landmark identification on monitor-displayed lateral cephalometric images. Am J Orthod Dentofacial Orthop 2008 Jun;133(6):790.e1-790e6.

7. Paixao MB, Sobral MC, Vogel CJ, Araujo TM. Comparative study between manual and digital cephalometric tracing using dolphin imaging software with lateral radiographs. Dent Press J Orthod 2010 Nov-Dec;15(6):123-130.

8. Shah AR, Karandikar G, Ravindranath VK, Sonawane M, Mhatre A. A comparative study of reliability and accuracy of manual and digital lateral cephalometric tracing. J Contemp Dent 2016 Jan-Apr;6(1):15-18.

9. Agarwal N, Bhagga DK, Sharma P. A comparative study of the cephalometric measurements with digital versus manual methods. J Ind Orthod Soc 2011 Apr;45(2):84-90.

10. McClure SR, Sadowaky PL, Ferreira A, Jacobson A. Reliability of digital versus conventional cephalometric radiology: a comparative evaluation of landmark identification error. Semin Orthod 2005 Jun;11(2):98-110.

11. Landis JR, Koch GG. The measurement of observer agreement for categorical data. Biometrics 1977;33:159-174.

12. Lim KF, Foong KW. Phosphor-stimulated computed cephalometry: reliability of landmark identification. Br J Orthod 1997 Dec;24(4):301-308.

13. Nikneshan S, Mohseni S, Nouri M, Hadian H, Kharazifard MJ. The effect of emboss enhancement on reliability of landmark identification in digital lateral cephalometric images. Iran J Radiol 2015 Apr;12(2):e19302.

14. Cooke MS, Wei SH. Cephalometric errors: a comparison between repeat measurements and retaken radiographs. Aust Dent J 1991 Feb;36(1):38-43.

15. Tikku T, Khanna R, Maurya RP, Srivastava K, Bhushan R. Comparative evaluation of cephalometric measurements of monitor-displayed images by Nemoceph software and its hard copy by manual tracing. J Oral Biol Craniofac Res 2014 Jan-Apr;4(1):35-41.

16. Dvortsin DP, Sandham A, Pruim GJ, Dijkstra PU. A comparison of the reproducibility of manual tracing and on-screen digitization for cephalometric profile variables. Eur J Orthod 2008 Dec;30(6):586-591. 